\title{
A statistical mechanical interpretation of algorithmic information theory III: Composite systems and fixed points
}

\author{
Kohtaro Tadaki \\ Research and Development Initiative, Chuo University \\ JST CREST \\ 1-13-27 Kasuga, Bunkyo-ku, Tokyo 112-8551, Japan \\ Email: tadaki@kc.chuo-u.ac.jp WWW: http://www2.odn.ne.jp/tadaki/
}

\begin{abstract}
The statistical mechanical interpretation of algorithmic information theory (AIT, for short) was introduced and developed by our former works [K. Tadaki, Local Proceedings of CiE 2008, pp. 425-434, 2008] and [K. Tadaki, Proceedings of LFCS'09, Springer's LNCS, vol. 5407, pp. 422-440, 2009], where we introduced the notion of thermodynamic quantities, such as partition function $Z(T)$, free energy $F(T)$, energy $E(T)$, and statistical mechanical entropy $S(T)$, into AIT. We then discovered that, in the interpretation, the temperature $T$ equals to the partial randomness of the values of all these thermodynamic quantities, where the notion of partial randomness is a stronger representation of the compression rate by means of program-size complexity. Furthermore, we showed that this situation holds for the temperature itself as a thermodynamic quantity, namely, for each of all the thermodynamic quantities above, the computability of its value at temperature $T$ gives a sufficient condition for $T \in(0,1)$ to be a fixed point on partial randomness. In this paper, we develop the statistical mechanical interpretation of AIT further and pursue its formal correspondence to normal statistical mechanics. The thermodynamic quantities in AIT are defined based on the halting set of an optimal computer, which is a universal decoding algorithm used to define the notion of program-size complexity. We show that there are infinitely many optimal computers which give completely different sufficient conditions in each of the thermodynamic quantities in AIT. We do this by introducing the notion of composition of computers into AIT, which corresponds to the notion of composition of systems in normal statistical mechanics.
\end{abstract}

\section{INTRODUCTION}

Algorithmic information theory (AIT, for short) is a framework for applying information-theoretic and probabilistic ideas to recursive function theory. One of the primary concepts of AIT is the program-size complexity (or Kolmogorov complexity) $H(s)$ of a finite binary string $s$, which is defined as the length of the shortest binary program for an optimal computer to output $s$. Here an optimal computer is a universal decoding algorithm. By the definition, $H(s)$ is thought to represent the amount of randomness contained in a finite binary string $s$, which cannot be captured in a computational manner. In particular, the notion of program-size complexity plays a crucial role in characterizing the randomness of an infinite binary string, or equivalently, a real.

In [12] we introduced and developed a statistical mechanical interpretation of AIT. We there introduced the notion of thermodynamic quantities at temperature $T$, such as partition function $Z(T)$, free energy $F(T)$, energy $E(T)$, and statistical mechanical entropy $S(T)$, into AIT. These quantities are real functions of a real argument $T>0$. We then proved that if the temperature $T$ is a computable real with $0<T<1$ then, for each of these thermodynamic quantities, the partial randomness of its value equals to $T$, where the notion of partial randomness is a stronger representation of the compression rate by means of program-size complexity. Thus, the temperature $T$ plays a role as the partial randomness of all the thermodynamic quantities in the statistical mechanical interpretation of AIT. In [12] we further showed that the temperature $T$ plays a role as the partial randomness of the temperature $T$ itself, which is a thermodynamic quantity of itself. Namely, we proved the fixed point theorem on partial randomness 1 which states that, for every $T \in(0,1)$, if the value of partition function $Z(T)$ at temperature $T$ is a computable real, then the partial randomness of $T$ equals to $T$, and therefore the compression rate of $T$ equals to $T$, i.e., $\lim _{n \rightarrow \infty} H\left(\left.T\right|_{n}\right) / n=T$, where $T \uparrow_{n}$ is the first $n$ bits of the base-two expansion of $T$.

In our second work [13] on this interpretation, we showed that a fixed point theorem of the same form as for $Z(T)$ holds also for each of free energy $F(T)$, energy $E(T)$, and statistical mechanical entropy $S(T)$. Moreover, based on the statistical mechanical relation $F(T)=-T \log _{2} Z(T)$, we showed that the computability of $F(T)$ gives completely different fixed points from the computability of $Z(T)$.

In this paper, we develop the statistical mechanical interpretation of AIT further and pursue its formal correspondence to normal statistical mechanics. As a result, we unlock the properties of the sufficient conditions further. The thermodynamic quantities in AIT are defined based on the halting set of an optimal computer. In this paper, we show in Theorem 9 below that there are infinitely many optimal computers which give completely different sufficient conditions in each of the

\footnotetext{
1 The fixed point theorem on partial randomness is called a fixed point theorem on compression rate in [12].
} 
thermodynamic quantities in AIT. We do this by introducing the notion of composition of computers into AIT, which corresponds to the notion of composition of systems in normal statistical mechanics.

\section{PReliminaries}

\section{A. Basic notation}

We start with some notation about numbers and strings which will be used in this paper. $\mathbb{N}=\{0,1,2,3, \ldots\}$ is the set of natural numbers, and $\mathbb{N}^{+}$is the set of positive integers. $\mathbb{Q}$ is the set of rationals, and $\mathbb{R}$ is the set of reals. Let $f: S \rightarrow \mathbb{R}$ with $S \subset \mathbb{R}$. We say that $f$ is increasing (resp., decreasing) if $f(x)<f(y)$ (resp., $f(x)>f(y)$ ) for all $x, y \in S$ with $x<y$.

Normally, $o(n)$ denotes any function $f: \mathbb{N}^{+} \rightarrow \mathbb{R}$ such that $\lim _{n \rightarrow \infty} f(n) / n=0$.

$\{0,1\}^{*}=\{\lambda, 0,1,00,01,10,11,000, \ldots\}$ is the set of finite binary strings, where $\lambda$ denotes the empty string. For any $s \in\{0,1\}^{*},|s|$ is the length of $s$. A subset $S$ of $\{0,1\}^{*}$ is called prefix-free if no string in $S$ is a prefix of another string in $S$. For any partial function $f$, the domain of definition of $f$ is denoted by $\operatorname{dom} f$.

Let $\alpha$ be an arbitrary real. We denote by $\left.\alpha\right|_{n} \in\{0,1\}^{*}$ the first $n$ bits of the base-two expansion of $\alpha-\lfloor\alpha\rfloor$ with infinitely many zeros, where $\lfloor\alpha\rfloor$ is the greatest integer less than or equal to $\alpha$. For example, in the case of $\alpha=5 / 8, \alpha\left\lceil_{6}=101000\right.$.

We say that a real $\alpha$ is computable if there exists a total recursive function $f: \mathbb{N}^{+} \rightarrow \mathbb{Q}$ such that $|\alpha-f(n)|<1 / n$ for all $n \in \mathbb{N}^{+}$. See e.g. Weihrauch [15] for the detail of the treatment of the computability of reals.

\section{B. Algorithmic information theory}

In the following we concisely review some definitions and results of algorithmic information theory [4], [5], [8], [7]. A computer is a partial recursive function $C:\{0,1\}^{*} \rightarrow\{0,1\}^{*}$ such that $\operatorname{dom} C$ is a nonempty prefix-free set. For each computer $C$ and each $s \in\{0,1\}^{*}, H_{C}(s)$ is defined by $H_{C}(s)=\min \left\{|p| \mid p \in\{0,1\}^{*} \& C(p)=s\right\}$ (may be $\infty$ ). A computer $U$ is said to be optimal if for each computer $C$ there exists $d \in \mathbb{N}$, which depends on $C$, with the following property; for every $p \in \operatorname{dom} C$ there exists $q \in\{0,1\}^{*}$ for which $U(q)=C(p)$ and $|q| \leq|p|+d$. It is easy to see that there exists an optimal computer. We choose a particular optimal computer $U$ as the standard one for use, and define $H(s)$ as $H_{U}(s)$, which is referred to as the program-size complexity of $s$ or the Kolmogorov complexity of $s$. It follows that for every computer $C$ there exists $d \in \mathbb{N}$ such that, for every $s \in\{0,1\}^{*}, H(s) \leq H_{C}(s)+d$.

For any $\alpha \in \mathbb{R}$, we say that $\alpha$ is weakly Chaitin random if there exists $c \in \mathbb{N}$ such that $n-c \leq H\left(\left.\alpha\right|_{n}\right)$ for all $n \in \mathbb{N}^{+}$ [4], [5]. On the other hand, for any $\alpha \in \mathbb{R}$, we say that $\alpha$ is Chaitin random if $\lim _{n \rightarrow \infty} H\left(\alpha \uparrow_{n}\right)-n=\infty$ [4], [5]. Obviously, for every $\alpha \in \mathbb{R}$, if $\alpha$ is Chaitin random, then $\alpha$ is weakly Chaitin random. We can show that the converse also hold. Thus, for every $\alpha \in \mathbb{R}, \alpha$ is weakly Chaitin random if and only if $\alpha$ is Chaitin random (see Chaitin [5] for the proof and historical detail).

\section{Partial randomness}

In the works [10], [11], we generalized the notion of the randomness of a real so that the degree of the randomness, which is often referred to as the partial randomness recently [2], [9], [3], can be characterized by a real $T$ with $0 \leq T \leq 1$ as follows.

Definition 1 (weak Chaitin $T$-randomness). Let $T \in \mathbb{R}$ with $T \geq 0$. For any $\alpha \in \mathbb{R}$, we say that $\alpha$ is weakly Chaitin $T$ random if there exists $c \in \mathbb{N}$ such that $T n-c \leq H\left(\left.\alpha\right|_{n}\right)$ for all $n \in \mathbb{N}^{+}$.

Definition 2 ( $T$-compressibility). Let $T \in \mathbb{R}$ with $T \geq 0$. For any $\alpha \in \mathbb{R}$, we say that $\alpha$ is $T$-compressible if $H\left(\left.\alpha\right|_{n}\right) \leq T n+$ $o(n)$, which is equivalent to $\lim _{\sup _{n \rightarrow \infty}} H\left(\left.\alpha\right|_{n}\right) / n \leq T$.

In the case of $T=1$, the weak Chaitin $T$-randomness results in the weak Chaitin randomness. For every $T \in[0,1]$ and every $\alpha \in \mathbb{R}$, if $\alpha$ is weakly Chaitin $T$-random and $T$ compressible, then

$$
\lim _{n \rightarrow \infty} \frac{H\left(\alpha \uparrow_{n}\right)}{n}=T .
$$

The left-hand side of (1) is referred to as the compression rate of a real $\alpha$ in general. Note, however, that (1) does not necessarily imply that $\alpha$ is weakly Chaitin $T$-random. Thus, the notion of partial randomness is a stronger representation of the notion of compression rate.

Definition 3 (Chaitin $T$-randomness, Tadaki [10], [11]). Let $T \in \mathbb{R}$ with $T \geq 0$. For any $\alpha \in \mathbb{R}$, we say that $\alpha$ is Chaitin T-random if $\lim _{n \rightarrow \infty} H\left(\left.\alpha\right|_{n}\right)-T n=\infty$.

In the case of $T=1$, the Chaitin $T$-randomness results in the Chaitin randomness. Obviously, for every $T \in[0,1]$ and every $\alpha \in \mathbb{R}$, if $\alpha$ is Chaitin $T$-random, then $\alpha$ is weakly Chaitin $T$-random. However, in 2005 Reimann and Stephan [9] showed that, in the case of $T<1$, the converse does not necessarily hold. This contrasts with the equivalence between the weak Chaitin randomness and the Chaitin randomness, each of which corresponds to the case of $T=1$.

\section{THE PREVIOUS RESULTS}

In this section, we review some results of the statistical mechanical interpretation of AIT, developed by our former works [12], [13]. We first introduce the notion of thermodynamic quantities into AIT in the following manner.

In statistical mechanics, the partition function $Z_{\mathrm{sm}}(T)$, free energy $F_{\mathrm{sm}}(T)$, energy $E_{\mathrm{sm}}(T)$, and entropy $S_{\mathrm{sm}}(T)$ at temperature $T$ are given as follows:

$$
\begin{aligned}
Z_{\mathrm{sm}}(T) & =\sum_{x \in X} e^{-\frac{E_{x}}{k_{\mathrm{B}} T}}, \\
F_{\mathrm{sm}}(T) & =-k_{\mathrm{B}} T \ln Z_{\mathrm{sm}}(T), \\
E_{\mathrm{sm}}(T) & =\frac{1}{Z_{\mathrm{sm}}(T)} \sum_{x \in X} E_{x} e^{-\frac{E_{x}}{k_{\mathrm{B}} T}}, \\
S_{\mathrm{sm}}(T) & =\frac{E_{\mathrm{sm}}(T)-F_{\mathrm{sm}}(T)}{T},
\end{aligned}
$$


where $X$ is a complete set of energy eigenstates of a quantum system and $E_{x}$ is the energy of an energy eigenstate $x$. The constant $k_{\mathrm{B}}$ is called the Boltzmann Constant, and the ln denotes the natural logarithm 2

Let $C$ be an arbitrary computer. We introduce the notion of thermodynamic quantities into AIT by performing Replacements 1 below for the thermodynamic quantities (2) in statistical mechanics.

\section{Replacements 1.}

(i) Replace the complete set $X$ of energy eigenstates $x$ by the set $\operatorname{dom} C$ of all programs $p$ for $C$.

(ii) Replace the energy $E_{x}$ of an energy eigenstate $x$ by the length $|p|$ of a program $p$.

(iii) Set the Boltzmann Constant $k_{\mathrm{B}}$ to $1 / \ln 2$.

Thus, motivated by the formulae (2) and taking into account Replacements 1, we introduce the notion of thermodynamic quantities into AIT as follows.

Definition 4 (thermodynamic quantities in AIT, [12]). Let $C$ be any computer, and let $T$ be any real with $T>0$.

First consider the case where $\operatorname{dom} C$ is an infinite set. In this case, we choose a particular enumeration $p_{1}, p_{2}, p_{3}, p_{4}, \ldots$ of the countably infinite set $\operatorname{dom} C \cdot 3$

(i) The partition function $Z_{C}(T)$ at temperature $T$ is defined as $\lim _{k \rightarrow \infty} Z_{k}(T)$ where

$$
Z_{k}(T)=\sum_{i=1}^{k} 2^{-\frac{\left|p_{i}\right|}{T}}
$$

(ii) The free energy $F_{C}(T)$ at temperature $T$ is defined as $\lim _{k \rightarrow \infty} F_{k}(T)$ where

$$
F_{k}(T)=-T \log _{2} Z_{k}(T) .
$$

(iii) The energy $E_{C}(T)$ at temperature $T$ is defined as $\lim _{k \rightarrow \infty} E_{k}(T)$ where

$$
E_{k}(T)=\frac{1}{Z_{k}(T)} \sum_{i=1}^{k}\left|p_{i}\right| 2^{-\frac{\left|p_{i}\right|}{T}} .
$$

(iv) The statistical mechanical entropy $S_{C}(T)$ at temperature $T$ is defined as $\lim _{k \rightarrow \infty} S_{k}(T)$ where

$$
S_{k}(T)=\frac{E_{k}(T)-F_{k}(T)}{T} .
$$

In the case where $\operatorname{dom} C$ is a nonempty finite set, the quantities $Z_{C}(T), F_{C}(T), E_{C}(T)$, and $S_{C}(T)$ are just defined as (3), (4), (5), and (6), respectively, where $p_{1}, \ldots, p_{k}$ is an enumeration of the finite set $\operatorname{dom} C$.

Note that, for every optimal computer $V, Z_{V}(1)$ is precisely a Chaitin $\Omega$ number introduced by Chaitin [4]. Theorems 5 ]and 6 below hold for these thermodynamic quantities in AIT.

\footnotetext{
${ }^{2}$ For the thermodynamic quantities in statistical mechanics, see e.g. Chapter 16 of [1] and Chapter 2 of [14]. To be precise, the partition function is not a thermodynamic quantity but a statistical mechanical quantity.

${ }^{3}$ The enumeration $\left\{p_{i}\right\}$ can be chosen quite arbitrarily, and the results of this paper are independent of the choice of $\left\{p_{i}\right\}$. This is because the sum $\sum_{i=1}^{k} 2^{-\left|p_{i}\right| / T}$ and $\sum_{i=1}^{k}\left|p_{i}\right| 2^{-\left|p_{i}\right| / T}$ in Definition 4 are positive term series and converge as $k \rightarrow \infty$ for every $T \in(0,1)$.
}

Theorem 5 (properties of $Z(T)$ and $F(T)$, [10], [11], [12]). Let $V$ be an optimal computer, and let $T \in \mathbb{R}$.

(i) If $0<T \leq 1$ and $T$ is computable, then each of $Z_{V}(T)$ and $F_{V}(T)$ converges and is weakly Chaitin T-random and $T$-compressible.

(ii) If $1<T$, then $Z_{V}(T)$ and $F_{V}(T)$ diverge to $\infty$ and $-\infty$, respectively.

Theorem 6 (properties of $E(T)$ and $S(T)$, [12]). Let $V$ be an optimal computer, and let $T \in \mathbb{R}$.

(i) If $0<T<1$ and $T$ is computable, then each of $E_{V}(T)$ and $S_{V}(T)$ converges and is Chaitin T-random and $T$ compressible.

(ii) If $1 \leq T$, then both $E_{V}(T)$ and $S_{V}(T)$ diverge to $\infty$.

The above two theorems show that if $T$ is a computable real with $T \in(0,1)$ then the temperature $T$ equals to the partial randomness (and therefore the compression rate) of the values of all the thermodynamic quantities in Definition 4 for an optimal computer.

These theorems also show that the values of all the thermodynamic quantities diverge when the temperature $T$ exceeds 1 . This phenomenon might be regarded as some sort of phase transition in statistical mechanics. Note here that the weak Chaitin $T$-randomness in Theorem 5 is replaced by the Chaitin $T$-randomness in Theorem 6 in exchange for the divergence at $T=1$.

In statistical mechanics or thermodynamics, among all thermodynamic quantities one of the most typical thermodynamic quantities is temperature itself. Theorem 7 below shows that the partial randomness of the temperature $T$ can equal to the temperature $T$ itself in the statistical mechanical interpretation of AIT.

We denote by $\mathcal{F} \mathcal{P}_{w}$ the set of all real $T \in(0,1)$ such that $T$ is weakly Chaitin $T$-random and $T$-compressible, and denote by $\mathcal{F P}$ the set of all real $T \in(0,1)$ such that $T$ is Chaitin $T$-random and $T$-compressible. Obviously, $\mathcal{F P} \subset \mathcal{F} \mathcal{P}_{w}$. Each element $T$ of $\mathcal{F} \mathcal{P}_{w}$ is a fixed point on partial randomness, i.e., satisfies the property that the partial randomness of $T$ equals to $T$ itself, and therefore satisfies that $\lim _{n \rightarrow \infty} H\left(\left.T\right|_{n}\right) / n=T$. Let $V$ be a computer. We define the sets $\mathcal{Z}(V)$ by

$$
\mathcal{Z}(V)=\left\{T \in(0,1) \mid Z_{V}(T) \text { is computable }\right\} .
$$

In the same manner, we define the sets $\mathcal{F}(V), \mathcal{E}(V)$, and $\mathcal{S}(V)$ based on the computability of $F_{V}(T), E_{V}(T)$, and $S_{V}(T)$, respectively. We can then show the following.

Theorem 7 (fixed points on partial randomness, [12], [13]). Let $V$ be an optimal computer. Then $\mathcal{Z}(V) \cup \mathcal{F}(V) \subset \mathcal{F} \mathcal{P}_{w}$ and $\mathcal{E}(V) \cup \mathcal{S}(V) \subset \mathcal{F P}$.

Theorem 7 is just a fixed point theorem on partial randomness, where the computability of each of the values $Z_{V}(T)$, $F_{V}(T), E_{V}(T)$, and $S_{V}(T)$ gives a sufficient condition for a real $T \in(0,1)$ to be a fixed point on partial randomness. Thus, by Theorem 7 , the above observation that the temperature $T$ equals to the partial randomness of the values of the thermo- 
dynamic quantities in the statistical mechanical interpretation of AIT is further confirmed.

\section{THE MAIN RESULT}

In this paper, we investigate the properties of the sufficient conditions for $T$ to be a fixed point on partial randomness in Theorem 7 Using the monotonicity and continuity of the functions $Z_{V}(T)$ and $F_{V}(T)$ on temperature $T$ and using the statistical mechanical relation $F_{V}(T)=-T \log _{2} Z_{V}(T)$, which holds from Definition 4 , we can show the following theorem for the sufficient conditions in Theorem 7

Theorem 8 ([13]). Let $V$ be an optimal computer. Then each of the sets $\mathcal{Z}(V)$ and $\mathcal{F}(V)$ is dense in $(0,1)$ while $\mathcal{Z}(V) \cap$ $\mathcal{F}(V)=\emptyset$.

Thus, for every optimal computer $V$, the computability of $F_{V}(T)$ gives completely different fixed points from the computability of $Z_{V}(T)$. This implies also that $\mathcal{Z}(V) \varsubsetneqq \mathcal{F} \mathcal{P}_{w}$ and $\mathcal{F}(V) \varsubsetneqq \mathcal{F} \mathcal{P}_{w}$.

The aim of this paper is to investigate the structure of $\mathcal{F} \mathcal{P}_{w}$ and $\mathcal{F P}$ in greater detail. Namely, we show in Theorem 9 below that there are infinitely many optimal computers which give completely different sufficient conditions in each of the thermodynamic quantities in AIT. We say that an infinite sequence $V_{1}, V_{2}, V_{3}, \ldots$ of computers is recursive if there exists a partial recursive function $F: \mathbb{N}^{+} \times\{0,1\}^{*} \rightarrow\{0,1\}^{*}$ such that for each $n \in \mathbb{N}^{+}$the following two hold: (i) $p \in \operatorname{dom} V_{n}$ if and only if $(n, p) \in \operatorname{dom} F$, and (ii) $V_{n}(p)=F(n, p)$ for every $p \in \operatorname{dom} V_{n}$. Then the main result of this paper is given as follows.

Theorem 9 (main result). There exists a recursive infinite sequence $V_{1}, V_{2}, V_{3}, \ldots$ of optimal computers which satisfies the following conditions:

(i) $\mathcal{Z}\left(V_{i}\right) \cap \mathcal{Z}\left(V_{j}\right)=\mathcal{F}\left(V_{i}\right) \cap \mathcal{F}\left(V_{j}\right)=\mathcal{E}\left(V_{i}\right) \cap \mathcal{E}\left(V_{j}\right)=$ $\mathcal{S}\left(V_{i}\right) \cap \mathcal{S}\left(V_{j}\right)=\emptyset$ for all $i, j$ with $i \neq j$.

(ii) $\bigcup_{i} \mathcal{Z}\left(V_{i}\right) \subset \mathcal{F} \mathcal{P}_{w}$ and $\bigcup_{i} \mathcal{F}\left(V_{i}\right) \subset \mathcal{F} \mathcal{P}_{w}$.

(iii) $\bigcup_{i} \mathcal{E}\left(V_{i}\right) \subset \mathcal{F P}$ and $\bigcup_{i} \mathcal{S}\left(V_{i}\right) \subset \mathcal{F P}$.

In the subsequent sections we prove the above theorems by introducing the notion of composition of computers into AIT, which corresponds to the notion of composition of systems in normal statistical mechanics.

\section{COMPOSITION OF COMPUTERS}

Definition 10 (composition of computers).

Let $C_{1}, C_{2}, \ldots, C_{N}$ be computers. The composition $C_{1} \oslash C_{2} \oslash$ $\cdots \oslash C_{N}$ of $C_{1}, C_{2}, \ldots$, and $C_{N}$ is defined as the computer $D$ such that (i) $\operatorname{dom} D=\left\{p_{1} p_{2} \ldots p_{N} \mid p_{1} \in \operatorname{dom} C_{1} \& p_{2} \in\right.$ $\left.\operatorname{dom} C_{2} \& \cdots \& p_{N} \in \operatorname{dom} C_{N}\right\}$, and (ii) $D\left(p_{1} p_{2} \ldots p_{N}\right)=$ $C_{1}\left(p_{1}\right)$ for every $p_{1} \in \operatorname{dom} C_{1}, p_{2} \in \operatorname{dom} C_{2}, \ldots$, and $p_{N} \in$ $\operatorname{dom} C_{N}$.

Theorem 11. Let $C_{1}, C_{2}, \ldots, C_{N}$ be computers. If $C_{1}$ is optimal then $C_{1} \oslash C_{2} \oslash \cdots \oslash C_{N}$ is also optimal.

Proof: We first choose particular strings $r_{2}, r_{3}, \ldots, r_{N}$ with $r_{2} \in \operatorname{dom} C_{2}, r_{3} \in \operatorname{dom} C_{3}, \ldots$, and $r_{N} \in \operatorname{dom} C_{N}$.
Let $C$ be an arbitrary computer. Then, by the definition of the optimality of $C_{1}$, there exists $d \in \mathbb{N}$ with the following property; for every $p \in \operatorname{dom} C$ there exists $q \in\{0,1\}^{*}$ for which $C_{1}(q)=C(p)$ and $|q| \leq|p|+d$. It follows from the definition of the composition $C_{1} \oslash C_{2} \oslash \cdots \oslash C_{N}$ that for every $p \in \operatorname{dom} C$ there exists $q \in\{0,1\}^{*}$ for which $\left(C_{1} \oslash\right.$ $\left.C_{2} \oslash \cdots \oslash C_{N}\right)\left(q r_{2} r_{3} \ldots r_{N}\right)=C(p)$ and $\left|q r_{2} r_{3} \ldots r_{N}\right| \leq$ $|p|+\left|r_{2} r_{3} \ldots r_{N}\right|+d$. Thus $C_{1} \oslash C_{2} \oslash \cdots \oslash C_{N}$ is an optimal computer.

In the same manner as in normal statistical mechanics, we can prove Theorem 12 below for the thermodynamic quantities in AIT. In particular, the equations (7), (8), and (9) correspond to the fact that free energy, energy, and entropy are extensive parameters in thermodynamics, respectively.

Theorem 12. Let $C_{1}, C_{2}, \ldots, C_{N}$ be computers. Then the following hold for every $T \in(0,1)$.

$$
\begin{aligned}
Z_{C_{1} \oslash \cdots \oslash C_{N}}(T) & =Z_{C_{1}}(T) \cdots Z_{C_{N}}(T), \\
F_{C_{1} \oslash \cdots \oslash C_{N}}(T) & =F_{C_{1}}(T)+\cdots+F_{C_{N}}(T), \\
E_{C_{1} \oslash \cdots \oslash C_{N}}(T) & =E_{C_{1}}(T)+\cdots+E_{C_{N}}(T), \\
S_{C_{1} \oslash \cdots \oslash C_{N}}(T) & =S_{C_{1}}(T)+\cdots+S_{C_{N}}(T) .
\end{aligned}
$$

For any computer $C$ and any $n \in \mathbb{N}^{+}$, the computer $\underbrace{C \oslash \cdots \oslash C}_{n}$ is denoted by $C^{\oslash n}$.

\section{THE PROOF OF THE MAIN RESULT}

In order to prove the main result, Theorem 9, we also introduce the notion of physically reasonable computer.

Definition 13 (physically reasonable computer). For any computer $C$, we say that $C$ is physically reasonable if there exist $p, q \in \operatorname{dom} C$ such that $|p| \neq|q|$.

Then we can prove Theorem 14 below in a similar manner to the proof of Theorem 7 of [13].

Theorem 14. Let $C$ be a physically reasonable computer. Then each of the mapping $(0,1) \ni T \mapsto Z_{C}(T)$, the mapping $(0,1) \ni T \mapsto E_{C}(T)$, and the mapping $(0,1) \ni T \mapsto S_{C}(T)$ is an increasing real function. On the other hand, the mapping $(0,1) \ni T \mapsto F_{C}(T)$ is a decreasing real function.

In order to prove the main result, it is also convenient to use the notion of computable measure machine, which was introduced by Downey and Griffiths [6] in 2004 for the purpose of characterizing the notion of Schnorr randomness of a real in terms of program-size complexity.

Definition 15 (computable measure machine, [6]). A computer $C$ is called a computable measure machine if $\sum_{p \in \operatorname{dom} C} 2^{-|p|}$ (i.e. $Z_{C}(1)$ ) is computable.

For the thermodynamic quantities in AIT, we can prove Theorem 16 below using Theorem 14

Theorem 16. Let $C$ be a physically reasonable, computable measure machine. Then, for every $T \in(0,1)$, the following conditions are equivalent: 
(i) $T$ is computable.

(ii) One of $Z_{C}(T), F_{C}(T), E_{C}(T)$, and $S_{C}(T)$ is computable.

(iii) All of $Z_{C}(T), F_{C}(T), E_{C}(T)$, and $S_{C}(T)$ are computable.

Example 17. The following two computers are examples of physically reasonable, computable measure machines.

(i) Two level system: Let $B$ be a particular computer for which $\operatorname{dom} B=\{1,01\}$. Then we see that, for every $T>0$,

$$
\begin{aligned}
Z_{B}(T) & =2^{-1 / T}+2^{-2 / T}, \\
F_{B}(T) & =-T \log _{2} Z_{B}(T), \\
E_{B}(T) & =\frac{1}{Z_{B}(T)}\left(2^{-1 / T}+2 \cdot 2^{-2 / T}\right), \\
S_{B}(T) & =\left(E_{B}(T)-F_{B}(T)\right) / T .
\end{aligned}
$$

(ii) One dimensional harmonic oscillator: Let $O$ be a particular computer for which $\operatorname{dom} O=\left\{0^{l} 1 \mid l \in \mathbb{N}\right\}$. Then we see that, for every $T>0$,

$$
\begin{aligned}
Z_{O}(T) & =\frac{1}{2^{1 / T}-1}, \\
F_{O}(T) & =T \log _{2}\left(2^{1 / T}-1\right), \\
E_{O}(T) & =\frac{2^{1 / T}}{2^{1 / T}-1}, \\
S_{O}(T) & =\left(E_{O}(T)-F_{O}(T)\right) / T .
\end{aligned}
$$

Since $Z_{B}(1)=3 / 4$ and $Z_{O}(1)=1$, we see that $B$ and $O$ are physically reasonable, computable measure machines. Note that Theorems 14 and 16 certainly hold for each of the particular physically reasonable computers $B$ and $O$.

Based on Theorems 12 and 16 , the main result is proved as follows.

The proof of Theorem 9. We first choose any optimal computer $V$ and any physically reasonable, computable measure machine $C$. Then, for each $n \in \mathbb{N}^{+}$, we denote the computer $V \oslash\left(C^{\oslash n}\right)$ by $V_{n}$. By Theorem 11, we see that $V_{n}$ is optimal for every $n \in \mathbb{N}^{+}$. Furthermore, it is easy to see that the infinite sequence $V_{1}, V_{2}, V_{3}, \ldots$ of computers is recursive. On the other hand, it follows from Theorem 12 that, for every $n \in \mathbb{N}^{+}$and every $T \in(0,1)$,

$$
\begin{aligned}
& Z_{V_{n}}(T)=Z_{V}(T) Z_{C}(T)^{n}, \\
& F_{V_{n}}(T)=F_{V}(T)+n F_{C}(T), \\
& E_{V_{n}}(T)=E_{V}(T)+n E_{C}(T), \\
& S_{V_{n}}(T)=S_{V}(T)+n S_{C}(T) .
\end{aligned}
$$

Let $m$ and $n$ be arbitrary two positive integers with $m>n$. Then it follows from the equations (10) that

$$
\begin{aligned}
Z_{V_{m}}(T) & =Z_{V_{n}}(T) Z_{C}(T)^{m-n}, \\
F_{V_{m}}(T) & =F_{V_{n}}(T)+(m-n) F_{C}(T), \\
E_{V_{m}}(T) & =E_{V_{n}}(T)+(m-n) E_{C}(T), \\
S_{V_{m}}(T) & =S_{V_{n}}(T)+(m-n) S_{C}(T)
\end{aligned}
$$

for every $T \in(0,1)$. In what follows, using (12) we show that $\mathcal{F}\left(V_{m}\right) \cap \mathcal{F}\left(V_{n}\right)=\emptyset$. In a similar manner, using (11), (13), and (14) we can show that $\mathcal{Z}\left(V_{m}\right) \cap \mathcal{Z}\left(V_{n}\right)=\mathcal{E}\left(V_{m}\right) \cap \mathcal{E}\left(V_{n}\right)=$ $\mathcal{S}\left(V_{m}\right) \cap \mathcal{S}\left(V_{n}\right)=\emptyset$ as well.

Now, let us assume contrarily that $\mathcal{F}\left(V_{m}\right) \cap \mathcal{F}\left(V_{n}\right) \neq \emptyset$. Then there exists $T_{0} \in(0,1)$ such that both $F_{V_{m}}\left(T_{0}\right)$ and $F_{V_{n}}\left(T_{0}\right)$ are computable. It follows from (12) that

$$
F_{C}\left(T_{0}\right)=\frac{1}{m-n}\left(F_{V_{m}}\left(T_{0}\right)-F_{V_{n}}\left(T_{0}\right)\right) .
$$

Thus, $F_{C}\left(T_{0}\right)$ is also computable. Since $C$ is a physically reasonable, computable measure machine, it follows from Theorem 16 that $T_{0}$ is also computable. Therefore, since $V_{m}$ is optimal, it follows from Theorem 5 (i) that $F_{V_{m}}\left(T_{0}\right)$ is weakly Chaitin $T_{0}$-random. However, this contradicts the fact that $F_{V_{m}}\left(T_{0}\right)$ is computable. Thus we have $\mathcal{F}\left(V_{m}\right) \cap \mathcal{F}\left(V_{n}\right)=\emptyset$. This completes the proof of Theorem 9 (i).

Theorem 9 (ii) and Theorem 9 (iii) follow immediately from Theorem 7 and the fact that $V_{i}$ is optimal for all $i \in \mathbb{N}^{+}$.

\section{ACKNOWLEDGMENTS}

This work was supported by KAKENHI, Grant-in-Aid for Scientific Research (C) (20540134), by SCOPE of the Ministry of Internal Affairs and Communications of Japan, and by CREST of the Japan Science and Technology Agency.

\section{REFERENCES}

[1] H. B. Callen, Thermodynamics and an Introduction to Thermostatistics, 2nd ed. John Wiley \& Sons, Inc., Singapore, 1985.

[2] C. S. Calude, L. Staiger, and S. A. Terwijn, "On partial randomness," Ann. Pure Appl. Logic, vol. 138, pp. 20-30, 2006.

[3] C. S. Calude and M. A. Stay, "Natural halting probabilities, partial randomness, and zeta functions," Inform. and Comput., vol. 204, pp. 17181739, 2006.

[4] G. J. Chaitin, "A theory of program size formally identical to information theory," J. Assoc. Comput. Mach., vol. 22, pp. 329-340, 1975.

[5] G. J. Chaitin, Algorithmic Information Theory. Cambridge University Press, Cambridge, 1987.

[6] R. G. Downey and E. J. Griffiths, "Schnorr randomness," J. Symbolic Logic, vol. 69, pp. 533-554, 2004.

[7] R. G. Downey and D. R. Hirschfeldt, Algorithmic Randomness and Complexity. Springer-Verlag, To appear.

[8] A. Nies, Computability and Randomness. Oxford University Press Inc., New York, 2009.

[9] J. Reimann and F. Stephan, On hierarchies of randomness tests. Proceedings of the 9th Asian Logic Conference, World Scientific Publishing, August 16-19, 2005, Novosibirsk, Russia.

[10] K. Tadaki, Algorithmic information theory and fractal sets. Proceedings of 1999 Workshop on Information-Based Induction Sciences (IBIS'99), pp. 105-110, August 26-27, 1999, Syuzenji, Shizuoka, Japan. In Japanese.

[11] K. Tadaki, "A generalization of Chaitin's halting probability $\Omega$ and halting self-similar sets," Hokkaido Math. J., vol. 31, pp. 219-253, 2002

[12] K. Tadaki, A statistical mechanical interpretation of algorithmic information theory. Local Proceedings of Computability in Europe 2008 (CiE 2008), pp. 425-434, June 15-20, 2008, University of Athens, Greece. Extended and Electronic Version Available: http://arxiv.org/abs/0801.4194v1

[13] K. Tadaki, Fixed point theorems on partial randomness. Proceedings of the Symposium on Logical Foundations of Computer Science 2009 (LFCS'09), Lecture Notes in Computer Science, Springer-Verlag, vol. 5407, pp. 422-440, 2009.

[14] M. Toda, R. Kubo, and N. Saitô, Statistical Physics I. Equilibrium Statistical Mechanics, 2nd ed. Springer, Berlin, 1992.

[15] K. Weihrauch, Computable Analysis. Springer-Verlag, Berlin, 2000. 